\title{
AVAliação de Estratégias PaRa REDUÇÃo da DERIVA dE AGRotóxicos EM PULVERIZAÇÕEs HIDRÁUliCAS ${ }^{1}$
}

\author{
Evaluation of Strategies to Reduce Pesticide Spray Drift \\ CUNHA, J.P.A.R. ${ }^{2}$, TEIXEIRA, M.M. ${ }^{3}$, COURY, J.R. ${ }^{4}$ e FERREIRA, L.R. ${ }^{5}$
}

\begin{abstract}
RESUMO - A deriva nas aplicações de agrotóxicos é considerada um dos maiores problemas da agricultura. Entre os fatores que a influenciam, o tamanho das gotas pulverizadas tem-se mostrado primordial. Dessa forma, o presente trabalho teve como objetivo a avaliação dos efeitos da adição de óleo vegetal emulsionável à calda de pulverização e do uso de pontas antideriva sobre o espectro de gotas e sobre o potencial de deriva de bicos de pulverização hidráulicos de jato plano. Em ambiente controlado, avaliou-se a população de gotas, por meio de um analisador de gotas por difração de raio laser, em tempo real, pulverizadas utilizandose bicos standard, com e sem a adição de adjuvante, e bicos antideriva, na faixa de pressão de 200 a $400 \mathrm{kPa}$. Como complementação, avaliou-se também a deriva em campo, utilizando-se alvos artificiais fora da área de aplicação, procedendo-se à contagem de gotas em diferentes distâncias. Observou-se que a adição de óleo vegetal à calda de pulverização e o uso de bicos de pulverização antideriva, dotados de pré-orifício, alteraram o espectro de gotas pulverizadas, aumentando o diâmetro das gotas e diminuindo a percentagem de gotas propensas à ação dos ventos, constituindo-se, portanto, em fator auxiliar para redução da deriva.
\end{abstract}

Palavras-chave: tecnologia de aplicação, bicos de pulverização, adjuvantes, gotas.

ABSTRACT - Pesticide spray drift is a major problem in agriculture today. Among the factors influencing drift, droplet size is of paramount importance. Thus, this work aimed to evaluate the effects of adding emulsifying vegetable oil to the pesticide emulsion and using low drift nozzles on droplet spectrum formation and, consequently, on the drift potential of fan spray nozzles. Droplet distribution was measured under controlled atmosphere, using a real time laser particle size analyzer. The droplets were generated with standard nozzles, with and without the addition of adjuvants, and with low drift nozzles, with the operating pressure ranging from 200 to 400 $\mathrm{kPa}$. Also, drift was evaluated in the field, using artificial targets placed outside the application area, where droplet counting was accomplished. The results showed that both the addition of vegetable oil to the pesticide emulsion and the use of low drift nozzles altered droplet size spectra, increasing the diameter of the drops and reducing the percentage of drops subject to wind action being, thus, effective factors in drift reduction.

Key words: application technology, nozzles, adjuvants, and droplets.

\section{INTRODUÇÃO}

Os agrotóxicos, embora desempenhem papel de fundamental importância dentro do sistema de produção agrícola vigente, têm sido alvo de crescente preocupação por parte dos diversos segmentos da sociedade, em virtude de seu potencial de risco ambiental (Barcellos et al., 1998). Tradicionalmente, há na sociedade consenso de que os agrotóxicos são prejudiciais ao homem, devido às notícias de contaminação de animais e seres humanos. No

Recebido para publicação em 26.11.2002 e na forma revisada em 11.8.2003.

2 Doutorando, Bolsista do CNPq, Departamento de Engenharia Agrícola da Universidade Federal de Viçosa - UFV, 36571-000 Viçosa-MG; ${ }^{3}$ Professor, Departamento de Engenharia Agrícola da UFV. ${ }^{4}$ Professor, Departamento de Engenharia Química, Universidade Federal de São Carlos - UFSC. ${ }^{5}$ Professor, Departamento de Fitotecnia da UFV. 
entanto, o seu uso tem contribuído para a prática agrícola, por meio da redução de mão-deobra e do aumento da produção, abaixando os custos e melhorando a qualidade dos alimentos. Sua utilização deve ser feita de maneira racional, dentro do contexto mais amplo da proteção integrada de plantas. Evitam-se, assim, a contaminação do solo e da água, os danos à saúde humana e animal e o aparecimento de pragas, doenças e plantas daninhas mais resistentes.

Dentre as diferentes técnicas de aplicação de agrotóxicos disponíveis, as que se baseiam na pulverização hidráulica são as mais difundidas, graças à flexibilidade que oferecem em distintas aplicações (Teixeira, 1997). Existem vários tipos de pulverizadores hidráulicos, que vão desde os mais simples, do tipo costal, utilizado em pequenas áreas, até os equipamentos mais sofisticados, como os pulverizadores de barra autopropelidos. Nesses equipamentos, os bicos de pulverização representam, sem dúvida, um dos principais componentes: garantem a qualidade e a segurança da aplicação (Tewari et al., 1998). O que se chama genericamente de bico é o conjunto de peças colocado no final do circuito hidráulico, através do qual a calda é emitida para fora da máquina. Esse conjunto é composto de várias partes, das quais a ponta de pulverização é a mais importante, regulando a vazão, o tamanho das gotas e a forma do jato emitido (Christofoletti, 1999).

Na maioria das vezes, dá-se muita importância ao produto fitossanitário a ser aplicado e pouca à técnica de aplicação. Não basta conhecer o produto a ser aplicado, também é fundamental conhecer a forma de aplicação. É preciso garantir que o produto alcance o alvo de forma eficiente, minimizando-se as perdas. Para isso, é necessário uniformidade de aplicação e espectro de gotas adequado.

O objetivo da tecnologia de aplicação é colocar a quantidade certa de ingrediente ativo no alvo desejado, com a máxima eficiência e da maneira mais econômica possivel, sem afetar o ambiente (Durigan, 1989). Muitas vezes, entretanto, parte do produto aplicado se perde no ambiente, principalmente por deriva. Além do prejuízo resultante da perda de agrotóxico e dos danos que podem ser causados em culturas adjacentes, a deriva assume grande importância devido à conscientização da população em relação à qualidade de água e alimentos e a outras questões ambientais (Lunkes, 1996).

A deriva é considerada um dos maiores problemas da agricultura (Sumner \& Sumner, 1999). O desvio da trajetória que impede que as gotas produzidas atinjam o alvo está relacionado, principalmente, ao tamanho de gotas e à velocidade do vento (Silva, 1999). De acordo com as condições ambientais, é preciso conhecer o espectro das gotas pulverizadas, de forma a adequar o seu tamanho, garantindo, ao mesmo tempo, eficácia biológica e segurança ambiental. Vários pesquisadores consideram que gotas menores que $100 \mu \mathrm{m}$ são facilmente carregadas pelo vento, sofrendo mais intensamente a ação dos fenômenos climáticos (Sumner, 1997; Murphy et al., 2000; Wolf, 2000).

O tamanho de gotas produzidas por um bico de pulverização depende de vários fatores, dentre os quais podem-se destacar as propriedades do líquido pulverizado e o tipo de bico. Fluidos com maior viscosidade e tensão superficial requerem maior quantidade de energia para a pulverização. Portanto, a pulverização de liquidos que tenham maior viscosidade $\mathrm{e}$ maior tensão superficial produz gotas maiores (Christofoletti, 1999). Dessa forma, uma opção economicamente viável encontrada para aumentar a viscosidade do líquido, bem como a eficiência das pulverizações, tem sido a adição de óleo vegetal às caldas de pulverização de herbicidas, fungicidas e inseticidas. A utilização de óleo vegetal como adjuvante tem indicação principal de espalhante adesivo, mas sua característica de viscosidade pode alterar também o espectro de gotas pulverizadas. As especificações dos bicos de pulverização são definidas em testes com água. O acréscimo de um adjuvante pode alterar o padrão de gotas e a vazão (Azevedo, 2001). Segundo Miller \& Butler Ellis (2000), mudanças nas propriedades do líquido pulverizado podem influenciar tanto o processo de formação das gotas como o comportamento destas em contato com o alvo, alterando o risco potencial de deriva da aplicação.

A escolha e o uso adequado de bicos de pulverização também constituem passos importantes para a melhoria das condições de 
precisão e segurança na aplicação de defensivos agrícolas (Womac et al., 1997). O conhecimento das condições de trabalho e, principalmente, do desempenho das pontas de pulverização são elementos básicos para uma aplicação adequada e eficiente (Christofoletti, 1999). De acordo com Johnson \& Swetnam (1996), a seleção apropriada dos bicos é essencial para a aplicação de defensivos, sendo o fator principal determinante da quantidade aplicada por área, da uniformidade de aplicação, da cobertura obtida e do risco potencial de deriva.

Existe no mercado uma série de bicos hidráulicos, em vários tipos e usos definidos para diferentes e específicas condições. Dentre os mais usados, destacam-se os de jato plano, que, dependendo das características de pressão e ângulo de abertura, podem apresentar alto risco de deriva. Os fabricantes, no entanto, têm lançado recentemente no mercado novos bicos, com grande potencial antideriva. Trata-se de pontas de pulverização dotadas de um préorificio, localizado antes da abertura para a formação do jato, que permite a formação de gotas de maior diâmetro.

Dessa forma, o presente trabalho teve como objetivo a avaliação dos efeitos da adição de óleo vegetal emulsionável à calda de pulverização e do uso de pontas antideriva sobre o espectro de gotas e potencial de deriva de bicos de pulverização hidráulicos de jato plano.

\section{MATERIAL E MÉTODOS}

O experimento foi realizado no Laboratório de Controle Ambiental do Departamento de Engenharia Química da Universidade Federal de São Carlos e no Laboratório de Mecanização Agrícola da Universidade Federal de Viçosa. Avaliou-se o espectro de gotas da pulverização hidráulica, utilizando-se bicos de jato plano standard, com e sem a adição de adjuvante à calda de pulverização, e bicos de jato plano antideriva, em três diferentes pressões. O adjuvante ésteres de ácidos graxos com glicerol (óleo vegetal emulsionável $93,0 \% \mathrm{v} / \mathrm{v}$ ), na formulação concentrado emulsionável, foi adicionado à calda de pulverização na dose de $0,5 \mathrm{~L}$ de produto comercial por $100 \mathrm{~L}$ de calda.

Utilizaram-se pontas de pulverização hidráulicas de jato plano, fabricadas em alumina revestida por poliacetal, adquiridas no mercado, com ângulo de abertura de $110^{\circ}$ e vazão nominal de $0,80 \mathrm{~L} \mathrm{~min}^{-1}$ à pressão de $275 \mathrm{kPa}$ : API 110-02 (Alumina Plastic ISO) e ADI 11002 (Alumina Drift ISO), fabricadas pela Albuz - Ceramiques Techniques Desmarquest, Evreux, France. Essas pontas de jato plano, de acordo com o fabricante, são de uso geral, produzindo aplicações uniformes quando se sobrepõem os jatos de pulverização, sendo recomendadas para trabalhar a pressões entre 200 e $400 \mathrm{kPa}$, com altura mínima da barra de $40 \mathrm{~cm}$ em relação ao alvo.

Em laboratório, a pulverização foi avaliada levando-se em conta o espectro de gotas produzidas e o potencial de deriva, considerando as pressões de 200, 300 e $400 \mathrm{kPa}$. Os parâmetros seguintes foram tomados: $\mathrm{D}_{\mathrm{v} 0,1}$ (diâmetro de gota tal que $10 \%$ do volume do líquido pulverizado é constituído de gotas de tamanho menor que esse valor), $\mathrm{D}_{\mathrm{v} 0,5}$ (diâmetro de gota tal que $50 \%$ do volume do líquido pulverizado é constituído de gotas de tamanho menor que esse valor, também conhecido como diâmetro da mediana volumétrica - DMV), $\mathrm{D}_{\mathrm{v} 0,9}$ (diâmetro de gota tal que $90 \%$ do volume do líquido pulverizado é constituído de gotas de tamanho menor que esse valor), SPAN (amplitude relativa) e percentagem do volume de gotas com diâmetro inferior a 100, 150 e $200 \mu \mathrm{m}$. Optouse pela determinação desses parâmetros, em vez da classificação em categorias sugerida pela ASAE (2000), com a finalidade de permitir maior distinção entre os tratamentos.

A amplitude relativa (SPAN) foi determinada utilizando-se a seguinte equação:

$$
S P A N=\frac{D_{v 0,9}-D_{v 0,1}}{D_{v 0,5}}
$$

O espectro da população de gotas foi determinado de forma direta, utilizando-se um analisador de gotas em tempo real, com base na técnica da difração de raio laser. Empregou-se o equipamento Spraytec (Malvern Spraytec Real Time Droplet Sizing System, Malvern Instruments, Malvern, England), com diâmetro do feixe do laser de $10 \mathrm{~mm}$, lente focal de $200 \mathrm{~mm}$, comprimento de onda do laser de $670 \mathrm{~nm}$ e ponte óptica de base longa. Inicialmente, verificou-se o alinhamento do feixe 
óptico, garantindo o seu correto posicionamento no sistema detector; monitorou-se o sistema de fundo (segundo plano) - medida da luz desviada no sistema óptico e da contaminação das janelas por partículas; e procedeuse à calibração do aparelho, utilizando-se o retículo-padrão RS-3 (retículo aprovado pelo ASTM - American Society for Testing and Materials - para calibração de instrumentos de análise de partículas baseados na técnica da difusão do laser). De acordo com o fabricante, o instrumento apresenta 3\% de acurácia e precisão de $1 \%$, utilizando-se o retículo-padrão. Para a análise dos dados, fez-se uso do software RTSizer ${ }^{\mathrm{TM}}$ (Insitec, Malvern, England).

$\mathrm{Na}$ determinação do espectro de gotas, utilizou-se um equipamento de pulverização costal, a pressão constante $\left(\mathrm{CO}_{2}\right)$, montado de tal forma que todo o jato pulverizado passasse transversalmente através do feixe luminoso do analisador, permitindo obter diretamente o espectro de gotas médio para cada condição desejada. O bico de pulverização situou-se a $25 \mathrm{~cm}$ acima do feixe óptico (ASAE, 2000).

Os ensaios foram realizados em ambiente controlado, com o intento de minimizar o efeito das condições ambientais (temperatura do ar inferior a $28^{\circ} \mathrm{C}$, umidade relativa do ar superior a $60 \%$ e ausência de ventos). Os manômetros utilizados foram previamente calibrados por meio de uma estrutura de reação dotada de massas-padrão, obtendo-se a relação entre pressão indicada e pressão real.

Como forma complementar, avaliou-se também a deriva dos três tratamentos em campo (jato plano standard, com e sem adjuvante, e jato plano antideriva), empregando-se a pressão de $300 \mathrm{kPa}$. Essa avaliação foi feita durante aplicações de fungicida em feijoeiro, fazendo-se uso do pulverizador costal $\left(\mathrm{CO}_{2}\right)$, a uma velocidade de deslocamento de $6 \mathrm{~km} \mathrm{~h}^{-1}$, perfazendo um volume de pulverização de $125 \mathrm{~L} \mathrm{ha}^{-1}$. Adotou-se altura do bico pulverizador de $50 \mathrm{~cm}$ em relação ao alvo.

Utilizaram-se alvos artificiais, etiquetas de papel sensivel à água (Ciba-Geigy), de acordo com metodologia apresentada por Wolf \& Frohberg (2002) e Sumner \& Sumner (1999). Os alvos foram coletados fora da área-alvo de aplicação, a 5, 10 e $15 \mathrm{~m}$ de distância da bordadura de aplicação, no sentido de deslocamento do vento, a um metro de altura. Durante as aplicações foram monitoradas a velocidade do vento, a umidade relativa e a temperatura do ar.

Esses papéis, disponíveis no mercado, são impregnados com o corante azul-de-bromofenol, que na sua forma não-ionizada apresenta coloração amarela. A água, entretanto, quando o atinge, ioniza a substância, e esta adquire forte coloração azul. Assim, após a aplicação, procedeu-se à contagem dos impactos ocorridos em cada etiqueta $(76 \times 26 \mathrm{~mm})$, sendo, portanto, indicativo da intensidade de deriva para cada tratamento. Os fabricantes do papel sensivel à água alertam para o fato de que esse substrato pode apresentar limitações de captura de gotas com diâmetro inferior a $30 \mu \mathrm{m}$. Essas gotas, no entanto, apresentam dificuldade de deposição em qualquer tipo de alvo (Chaim et al., 1999).

Para a análise estatística dos dados de espectro de gotas, considerou-se o experimento em delineamento inteiramente casualizado, com cinco repetições, no esquema fatorial $3 \times 3$ (três formas de aplicação e três pressões de operação). A análise estatística do estudo de deriva em campo, para as três formas de aplicação, foi feita considerando um delineamento inteiramente casualizado, com quatro repetições. Adotou-se o teste de Tukey, a 5\% de probabilidade, para o estudo comparativo das médias dos tratamentos. As análises estatísticas foram realizadas utilizando-se o programa estatístico Saeg 8.0.

\section{RESULTADOS E DISCUSSÃO}

A distribuição das gotas por classe de tamanho para a pulverização utilizando bicos de jato plano standard, com e sem a adição de adjuvante à calda, e bicos de jato plano antideriva, para as pressões de 200, 300 e $400 \mathrm{kPa}$, encontra-se na Figura 1. A inclinação da curva de volume acumulado indica a existência de gotas de diferentes tamanhos, razão pela qual essas pulverizações são consideradas de espectro heterogêneo. Uma pulverização que fosse formada por gotas do mesmo tamanho seria representada no gráfico por uma linha vertical (step function). Quanto mais inclinada a curva, maior será a variação do tamanho das gotas geradas (Christofoletti, 1999). 
A. Jato plano standard $-200 \mathrm{kPa}$

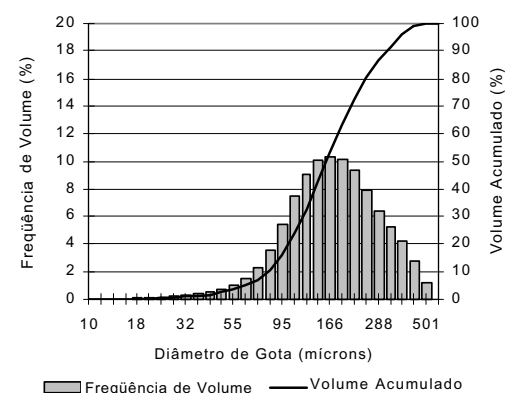

D. Jato plano standard com adjuvante $-200 \mathrm{kPa}$

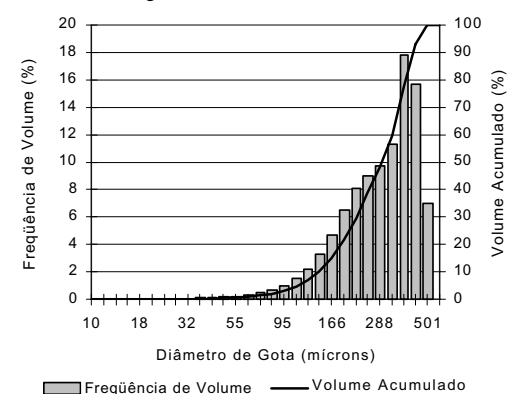

G. Jato plano antideriva $-200 \mathrm{kPa}$

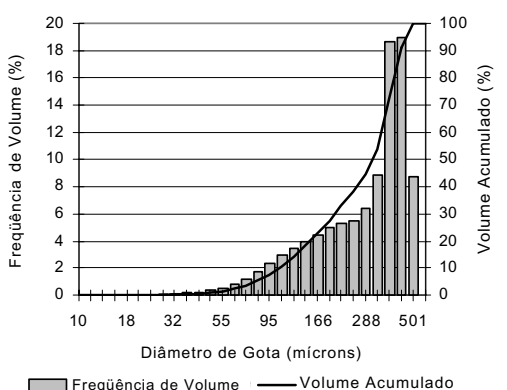

B. Jato plano standard $-300 \mathrm{kPa}$

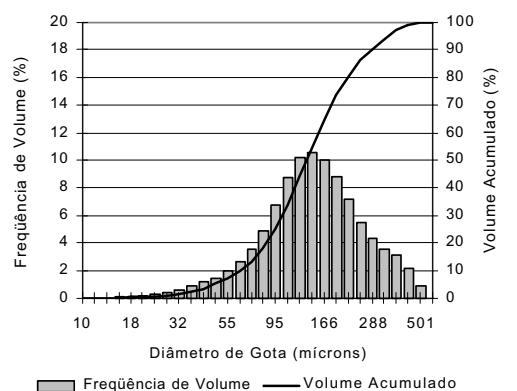

E. Jato plano standard com adjuvante $-300 \mathrm{kPa}$

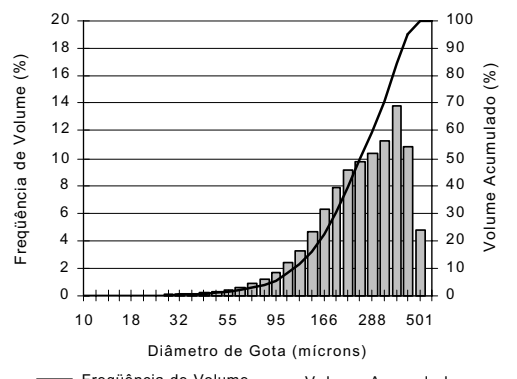

— Frequêencia de Volume — Volume Acumulado

H. Jato plano antideriva $-300 \mathrm{kPa}$

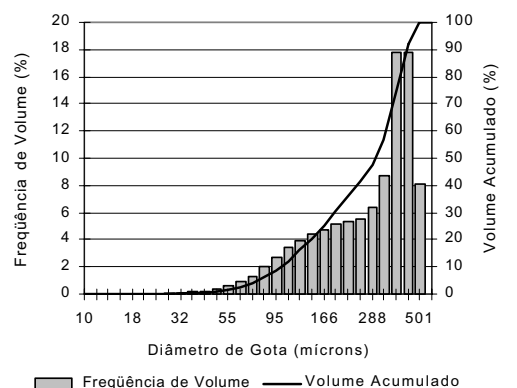

C. Jato plano standard $-400 \mathrm{kPa}$

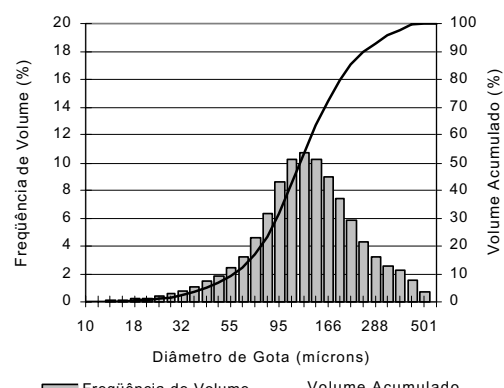

—Freqüência de Volume _ Volume Acumulado

F. Jato plano standard com adjuvante $-400 \mathrm{kPa}$

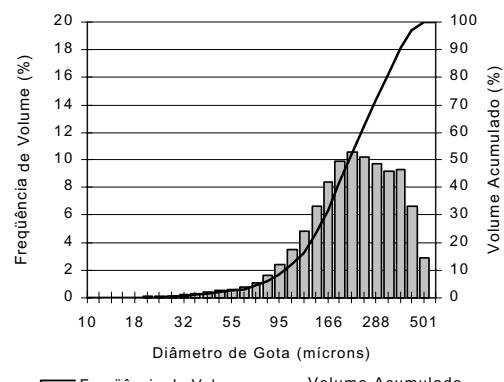

—reqüência de Volume _ Volume Acumulado

I. Jato plano antideriva $-400 \mathrm{kPa}$

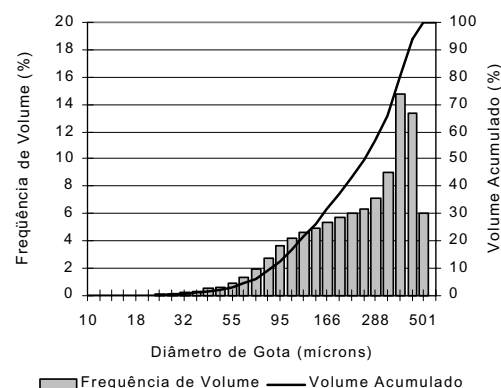

Figura 1 - Espectro de gotas pulverizadas utilizando bicos de jato plano standard (API 110-02), com e sem a adição de adjuvante à calda, e bicos de jato plano antideriva (ADI 110-02), para as pressões de 200, 300 e $400 \mathrm{kPa}$.

A Tabela 1 resume os valores dos diâmetros médios para os volumes acumulados de 10 , 50 e $90 \%$ e da amplitude relativa. Os diâmetros da mediana volumétrica (DMV) variaram de 124 a $322 \mu \mathrm{m}$. Em geral, valores de DMV inferiores a $250 \mu \mathrm{m}$ indicam risco potencial de deriva, que acontece principalmente em virtude das gotas menores que $100 \mu \mathrm{m}$. Em contrapartida, valores de DMV superiores a $500 \mu \mathrm{m}$ sugerem problemas de escorrimento, que, comumente, ocorrem com gotas maiores que $800 \mu \mathrm{m}$.

Na Tabela 2 é mostrada a percentagem do volume de gotas com diâmetro inferior a 100, 150 e $200 \mu \mathrm{m}$. Esses parâmetros indicam o potencial de deriva da aplicação. Quanto menores esses valores, menor o risco de perda de defensivo durante uma aplicação causada por arrastamento. Em geral, valores inferiores a $15 \%$ do volume pulverizado composto por gotas com diâmetro inferior a $100 \mu \mathrm{m}$ parecem ser mais adequados a uma aplicação segura.

Nota-se que os bicos de jato plano standard, avaliados sem a adição de óleo à calda, possuem alto potencial de risco de deriva, mesmo em baixa pressão. Em média, apresentaram DMV de $141 \mu \mathrm{m}$ e $24 \%$ do volume pulverizado composto por gotas com diâmetro inferior a $100 \mu \mathrm{m}$. 
Tabela 1 - Distribuição volumétrica por classe de tamanho e amplitude relativa de gotas pulverizadas utilizando bicos de jato plano standard, com e sem a adição de adjuvante à calda de pulverização, e bicos de jato plano antideriva, para as pressões de 200,300 e $400 \mathrm{kPa}$

\begin{tabular}{|c|c|c|c|c|c|c|c|c|c|c|c|c|}
\hline \multirow{3}{*}{ Tratamento } & \multicolumn{3}{|c|}{$\mathrm{D}_{\mathrm{v} 0,1}(\mu \mathrm{m})$} & \multicolumn{3}{|c|}{$\mathrm{D}_{\mathrm{v} 0,5}(\mu \mathrm{m})$} & \multicolumn{3}{|c|}{$\mathrm{D}_{\mathrm{v} 0,9}(\mu \mathrm{m})$} & \multicolumn{3}{|c|}{ SPAN } \\
\hline & \multicolumn{12}{|c|}{ Pressão (kPa) } \\
\hline & 200 & 300 & 400 & 200 & 300 & 400 & 200 & 300 & 400 & 200 & 300 & 400 \\
\hline $\begin{array}{l}\text { Bico standard*, } \\
\text { sem adjuvante }\end{array}$ & $81 \mathrm{Ac}$ & $64 \mathrm{Bc}$ & $57 \mathrm{Bb}$ & $164 \mathrm{Ac}$ & $134 \mathrm{Bc}$ & $124 \mathrm{Bc}$ & $330 \mathrm{Ab}$ & $280 \mathrm{Bb}$ & $256 \mathrm{Bc}$ & $1,52 \mathrm{Aa}$ & $1,61 \mathrm{Aa}$ & $1,60 \mathrm{Aa}$ \\
\hline $\begin{array}{l}\text { Bico standard*, } \\
\text { com adjuvante }\end{array}$ & $145 \mathrm{Aa}$ & $121 \mathrm{Ba}$ & $102 \mathrm{Ca}$ & $297 \mathrm{Ab}$ & $252 \mathrm{Bb}$ & $219 \mathrm{Cb}$ & $424 \mathrm{Aa}$ & $407 \mathrm{ABa}$ & $385 \mathrm{Bb}$ & $0,94 \mathrm{Bb}$ & $1,14 \mathrm{Ab}$ & $1,29 \mathrm{Ab}$ \\
\hline Bico antideriva** & $113 \mathrm{Ab}$ & $101 \mathrm{ABb}$ & $89 \mathrm{Ba}$ & $322 \mathrm{Aa}$ & $298 \mathrm{Ba}$ & $262 \mathrm{Ca}$ & 434Aa & 429Aa & $421 \mathrm{Aa}$ & $1,00 \mathrm{Bb}$ & $1,10 \mathrm{Bb}$ & $1,27 \mathrm{Ab}$ \\
\hline
\end{tabular}

* Bico API 110-02, ** Bico ADI 110-02

$\mathrm{D}_{\mathrm{v} 0,1}$ - diâmetro de gota tal que $10 \%$ do volume do líquido pulverizado é constituído de gotas de tamanho menor que esse valor; $\mathrm{D}_{\mathrm{v} 0,5}$ - diâmetro de gota tal que $50 \%$ do volume do líquido pulverizado é constituído de gotas de tamanho menor que esse valor; $\mathrm{D}_{\mathrm{v} 0,9}$ - diâmetro de gota tal que $90 \%$ do volume do líquido pulverizado é constituído de gotas de tamanho menor que esse valor; SPAN - amplitude relativa.

Médias seguidas pela mesma letra maiúscula, nas linhas, e minúscula, nas colunas, não diferem entre si a $5 \%$ de probabilidade pelo teste de Tukey.

Tabela 2 - Percentagem do volume pulverizado composto por gotas com diâmetro inferior a 100, 150 e $200 \mu \mathrm{m}$ utilizando bicos de jato plano standard, com e sem a adição de adjuvante à calda de pulverização, e bicos de jato plano antideriva, para as pressões de 200,300 e $400 \mathrm{kPa}$

\begin{tabular}{|c|c|c|c|c|c|c|c|c|c|}
\hline \multirow{3}{*}{ Tratamento } & \multicolumn{3}{|c|}{$\begin{array}{l}\% \text { Volume de Gotas } \\
\text { Menores que } 100 \mu \mathrm{m}\end{array}$} & \multicolumn{3}{|c|}{$\begin{array}{l}\% \text { Volume de Gotas } \\
\text { Menores que } 150 \mu \mathrm{m}\end{array}$} & \multicolumn{3}{|c|}{$\begin{array}{l}\% \text { Volume de Gotas } \\
\text { Menores que } 200 \mu \mathrm{m}\end{array}$} \\
\hline & \multicolumn{9}{|c|}{ Pressão $(\mathrm{kPa})$} \\
\hline & 200 & 300 & 400 & 200 & 300 & 400 & 200 & 300 & 400 \\
\hline $\begin{array}{l}\text { Bico } \text { standard*, } \\
\text { sem adjuvante }\end{array}$ & $15,71 \mathrm{Ca}$ & $25,57 \mathrm{Ba}$ & $30,72 \mathrm{Aa}$ & $40,97 \mathrm{Ca}$ & $55,70 \mathrm{Ba}$ & $61,68 \mathrm{Aa}$ & $60,97 \mathrm{Ba}$ & $74,33 \mathrm{Aa}$ & $79,04 \mathrm{Aa}$ \\
\hline $\begin{array}{l}\text { Bico standard*, } \\
\text { com adjuvante }\end{array}$ & $2,98 \mathrm{Cc}$ & $5,33 \mathrm{Bc}$ & $8,47 \mathrm{Ac}$ & $9,89 \mathrm{Cc}$ & $15,84 \mathrm{Bc}$ & $22,56 \mathrm{Ab}$ & $20,89 \mathrm{Cb}$ & $30,51 \mathrm{Bb}$ & $39,82 \mathrm{Ab}$ \\
\hline Bico antideriva** & $6,90 \mathrm{Bb}$ & $8,67 \mathrm{Bb}$ & $11,98 \mathrm{Ab}$ & $16,63 \mathrm{Bb}$ & $20,57 \mathrm{Bb}$ & $25,63 \mathrm{Ab}$ & $25,45 \mathrm{Bb}$ & $30,45 \mathrm{ABb}$ & $36,14 \mathrm{Ab}$ \\
\hline
\end{tabular}

* Bico API 110-02, ** Bico ADI 110-02

Médias seguidas pela mesma letra maiúscula, nas linhas, e minúscula, nas colunas, não diferem entre si a 5\% de probabilidade pelo teste de Tukey.

A adição de óleo vegetal à calda de pulverização aumentou o diâmetro das gotas. Comparando os diâmetros característicos dos bicos standard, com e sem adição de adjuvante, notase o aumento dos valores com o uso do adjuvante em todas as pressões estudadas. A percentagem de gotas com diâmetro inferior a 100 , 150 e $200 \mu \mathrm{m}$ diminuiu, demonstrando o potencial do adjuvante na redução da deriva. Esse resultado está em conformidade com os resultados apresentados por Cu et al. (1992). Esses autores, avaliando o efeito do óleo de soja como adjuvante no espectro de gotas analisadas em alvos artificiais, concluíram que ocorre aumento do diâmetro de gotas com o acréscimo de óleo à calda de pulverização. O óleo vegetal aumenta a tensão superficial e a viscosidade da calda a ser aplicada, dificultando sua desintegração em gotas pelo bico de pulverização (Sanderson et al., 1997). Também Wolf (2000) e Sumner (1997) mostram que a adição de adjuvantes à calda de pulverização pode alterar o espectro de gotas pulverizadas, reduzindo o risco potencial de deriva. 
Os bicos antideriva também contribuíram para o aumento do tamanho das gotas, diminuindo o risco potencial de deriva. O pré-orificio permite a formação de uma câmara, fazendo com que as gotas pequenas se juntem, formando gotas maiores. Utilizando bicos standard sem a adição de óleo, em média, 24\% do volume pulverizado apresentou gotas de diâmetro inferior a $100 \mu \mathrm{m}$; com a adição de 0,5 L de adjuvante por $100 \mathrm{~L}$ de calda, $6 \%$; e com a utilização de bicos antideriva, 9\%. À medida que se aumentou a pressão, o risco de deriva aumentou. Sumner \& Sumner (1999) também constataram a redução do potencial de deriva com a utilização de alguns bicos antideriva avaliados.

É possivel verificar que a amplitude relativa do tamanho das gotas (SPAN) foi mais favorável quando se trabalhou com bico standard com adição de óleo e bico antideriva. Os valores obtidos variaram de 0,94 a 1,61. À medida que se deseja aumentar a qualidade da pulverização, deve-se exigir mais do desempenho dos bicos e, especialmente, da homogeneidade do espectro de gotas. Numericamente, quanto maior o valor da amplitude relativa, maior será a faixa de tamanho das gotas pulverizadas. Espectro de gotas homogêneo tem valor de amplitude relativa tendendo a zero. Os valores de DMV e amplitude relativa devem ser analisados conjuntamente para a caracterização da pulverização. Isoladamente, o DMV fornece um valor de referência, sem indicar a dispersão dos dados em torno desse valor. A amplitude relativa indica a homogeneidade do tamanho das gotas.

Analisando os resultados, verifica-se também que a pressão influenciou o espectro de gotas. Maiores pressões ocasionaram menor tamanho de gotas. Portanto, uma maneira simples de se evitar a deriva consiste em trabalhar com os bicos de pulverização em pressões mais baixas, próximas ao limite inferior indicado pelos fabricantes para a boa uniformidade de distribuição dos bicos.

Na Tabela 3 é mostrado o resultado da avaliação em campo. As condições meteorológicas no momento das aplicações foram estáveis, com velocidade do vento variando entre o mínimo de 2,0 e o máximo de $2,5 \mathrm{~m} \mathrm{~s}^{-1}$, temperatura média de $21^{\circ} \mathrm{C}$ e umidade relativa em torno de $85 \%$. Em conformidade com os resultados apresentados anteriormente, os bicos standard
Tabela 3 - Densidade de gotas depositadas em alvos artificiais em diferentes distâncias da área-alvo, utilizando bicos de jato plano standard, com e sem a adição de adjuvante à calda de pulverização, e bicos de jato plano antideriva, à pressão de $300 \mathrm{kPa}$

\begin{tabular}{|l|c|c|c|}
\hline \multirow{2}{*}{ Tratamento } & \multicolumn{3}{|c|}{ Densidade de Gotas $\left(\right.$ gotas $\left.\mathrm{cm}^{-2}\right)$} \\
\cline { 2 - 4 } & \multicolumn{3}{|c|}{ Distância da Área-Alvo } \\
\cline { 2 - 4 } & $5 \mathrm{~m}$ & $10 \mathrm{~m}$ & $15 \mathrm{~m}$ \\
\hline $\begin{array}{l}\text { Bico Standard*, } \\
\text { sem adjuvante }\end{array}$ & $17,75 \mathrm{~A}$ & $7,75 \mathrm{~A}$ & $2,5 \mathrm{~A}$ \\
\hline $\begin{array}{l}\text { Bico Standard*, } \\
\text { com adjuvante }\end{array}$ & $3,00 \mathrm{~B}$ & $1,75 \mathrm{~B}$ & $0,75 \mathrm{~B}$ \\
\hline Bico antideriva** & $2,85 \mathrm{~B}$ & $1,25 \mathrm{~B}$ & $0,5 \mathrm{~B}$ \\
\hline
\end{tabular}

* Bico API 110-02, ** Bico ADI 110-02.

Médias seguidas pela mesma letra, nas colunas, não diferem entre si a $5 \%$ de probabilidade pelo teste de Tukey.

sem adjuvante apresentaram maior grau de deriva nas três distâncias avaliadas. As pontas antideriva e a adição de adjuvante permitiram controle da deriva, reduzindo o arrastamento de partículas e diminuindo significativamente o número de gotas fora da área-alvo.

Dessa forma, de acordo com a metodologia utilizada, pode-se concluir que a adição de óleo vegetal à calda de pulverização e o uso de bicos de pulverização antideriva dotados de pré-orificio alteraram o espectro de gotas pulverizadas, aumentando-lhes o diâmetro e diminuindo a percentagem daquelas gotas propensas à ação dos ventos, constituindo-se, portanto, em fator auxiliar para redução da deriva nas pulverizações.

\section{LITERATURA CITADA}

AMERICAN SOCIETY OF AGRICULTURAL ENGINEERING - ASAE. Spray nozzle classification by droplet spectra. St. Joseph: 2000. p. 389-391. (ASAE Standard, S572 AUG99).

AZEVEDO, L. A. S. Proteção integrada de plantas com fungicidas. São Paulo, 2001. 230 p.

BARCELlOS, L. C.; CARVALHO, Y. C.; SILVA, A. L. Estudo sobre a penetração de gotas de pulverização no dossel da cultura da soja [Glycine max (L.) Merrill]. Eng. Agric., v. 6, n. 2, p. 81-94, 1998.

CHAIM, A.; MAIA, A. H. N.; PESSOA, M. C. P. Y. Estimativa da deposição de agrotóxicos por análise de gotas. Pesq. Agropec. Bras., v. 34, n. 6, p. 963-969, 1999. 
CHRISTOFOLETTI, J. C. Considerações sobre a deriva nas pulverizações agrícolas e seu controle. São Paulo: Teejet South América, 1999. 15 p.

CU, R. M.; PHIPPS, P. M.; STIPES, R. J. Adjuvant effects of soyoil $937 \AA$ on fungicides for control of early leafspot and sclerotinia blight in peanuts. In: FOY, C. L. Adjuvants for agrichemicals. Boca Raton: CRC Press, 1992. p. 657666.

DURIGAN, J. C. Comportamento de herbicidas no ambiente. In: SEMINÁRIO TÉCNICO SOBRE PLANTAS DANINHAS E O USO DE HERBICIDAS EM REFLORESTAMENTO, 1989, Rio de Janeiro. Anais... Rio de Janeiro: SBS/ABRACAV/SIF, 1989. (paginaçao irregular)

JOHNSON, M. P.; SWETNAM, L. D. Sprayer nozzles: selection and calibration. Lexington: University of Kentucky, 1996. 6 p.

LUNKES, J. A. Efeito de subdoses de glyphosate e oxyfluorfen simulando deriva sobre a cultura do feijoeiro. 1996. $138 \mathrm{f}$. Tese (Doutorado em Agronomia) Universidade Federal de Lavras, Lavras, 1996.

MILLER, P. C. H.; BUTLER ELLIS, M. C. Effects of formulation on spray nozzle performance for applications from ground-based boom sprayers. Crop Prot., v. 19, p. 609-615, 2000.

MURPHY, S. D.; MILLER, P. C. H; PARKIN, C. S. The effect of boom section and nozzle configuration on the risk of spray drift. J. Agric. Eng. Res., v. 75, p. 127-137, 2000.

SANDERSON, R. et al. Relative drift potential and droplet size spectra of aerially applied propanil formulations. Crop Protec., v. 16, n. 8, p. 717-721, 1997.
SILVA, O. C. Tecnologia de aplicação de fungicidas. In: CANTERI, M. G.; PRIA, M. D.; SILVA, O. C. (Eds.) Principais doenças fúngicas do feijoeiro. Ponta Grossa: UEPG, 1999. p. 127-137.

SUMNER, P. E. Reducing spray drift. Georgia: University of Georgia, 1997. 11 p. (ENG97-005)

SUMNER, P. E.; SUMNER, S. A. Comparison of new drift reduction nozzles. St. Joseph: ASAE, 1999. 17 p. (ASAE Paper n.99-1156).

TEIXEIRA, M. M. Influencia del volumen de caldo y de la uniformidad de distribución transversal sobre la eficacia de la pulverización hidráulica. 1997. $310 \mathrm{f}$. Tese (Doutorado em Agronomia) - Escuela Técnica Superior de Ingenieros Agrônomos, Universidad Politécnica de Madrid, Madrid, 1997.

TEWARI, V. K.; MURALIKRISHNA, R. V. S.; PANDYA, A. C. Performance evaluation and computer aided design of valve type hollow cone nozzles. St. Joseph: ASAE, 1998. 15 p. (ASAE Paper n.98-1025).

WOLF, R. E. Strategies to reduce spray drift. Kansas: Kansas State University, 2000. 4 p. (Application Technology Series)

WOLF, R. E.; FROHBERG, D. D. Comparison of drift for four drift-reducing flat-fan nozzle types measured in a wind tunnel and evaluated using dropletscan software St. Joseph: ASAE, 2002. 7 p. (ASAE Paper n.02-1101)

WOMAC, A. R.; GOODWIN, J. C.; HART, W. E. Comprehensive evaluation of droplet spectra from drift reduction nozzles. St. Joseph: ASAE, 1997. 47 p. (ASAE Paper n.97-1069) 